population estimates and mortality data contemporaneous with the linked survey data.

Results There were 201 (6.4\%) male deaths and 215 (5.4\%) female deaths in the $2003 \mathrm{SHeS}$ by the end of 2008. Among men, all-cause mortality was markedly lower in the SHeS sample (918 per 100,000 person-years [95\% CI:850-987]) than in the Scottish population (1361 [95\% CI:1357-1365]). Figures for women were also highly significantly different (739 [95\% CI:682-795] for the SHeS and 928 [95\% CI:925-931] for the Scottish population). Alcohol-related mortality was lower in the SHeS sample (38 [95\% CI:18-57] in men and 11 [95\% CI:0-22] in women) relative to the Scottish population (57 [95\% CI:56-58] in men [non-significant] and 25 [95\% CI:24-25] in women [significant]).

Conclusion Respondents to the 2003 SHeS differ from the population they are intended to represent, with much lower than expected all-cause mortality in both sexes; alcohol-related mortality rates were somewhat lower than expected suggesting lower alcohol consumption among survey respondents which, if genuine, would lead to inherent underestimation of population consumption levels. Importantly, differences existed despite the application of conventional weighting and age-standardisation methods. Consideration should be given to the levels of resource allocated for increasing survey response and the further development of survey methodology to address the resultant systematic bias in health survey data arising from non-response.

\section{OP74 MEASURING SEXUAL BEHAVIOUR COMES OF AGE: A COMPARISON OF OUTCOMES IN THE 2010 HEALTH SURVEY FOR ENGLAND WITH THE NATIONAL SURVEY OF SEXUAL ATTITUDES AND LIFESTYLES}

doi:10.1136/jech-2012-201753.074

${ }^{1} \mathrm{CH}$ Mercer, ${ }^{2} \mathrm{C}$ Robinson, ${ }^{2} \mathrm{R}$ Craig, ${ }^{3} \mathrm{~A}$ Nardone, ${ }^{1} \mathrm{AM}$ Johnson. ${ }^{1}$ Infection \& Population Health, UCL, London, UK; ${ }^{2}$ Health \& Wellbeing Group , National Centre for Social Research, London, UK: ${ }^{3} \mathrm{HIV} / \mathrm{STI}$ Department, Health Protection Agency, London, UK

Background The Health Survey for England (HSE) is a general health survey administered annually to a probability sample of people living in England. In 2010, the HSE included, for the first time, questions about sexual health, which previously were considered too sensitive for a general health survey. This paper compares the reporting of sexual behaviours by people aged 16-44 in HSE-2010 with data collected by the second British National Survey of Sexual Attitudes and Lifestyles (Natsal-2), Britain's most recent, dedicated national probability survey of sexual behaviour.

Methods In HSE-2010, 8,420 people aged 16-69 were interviewed, of whom 2,911 were aged 16-44. Natsal-2 interviewed 12,110 people aged 16-44 in 1999/2001. HSE-2010 used pen-and-paper selfcompletion questionnaires for the sexual health questions, while Natsal-2 used computer-assisted personal-interviews including computer-assisted self-interview for the more sensitive questions, including those on sexual health. HSE-2010 used the same question wording developed and piloted for Natsal.

Results Collecting sexual behaviour data was acceptable to HSE2010 participants with low item non-response (5-10\%), albeit slightly higher than in Natsal-2 $(<5 \%)$. Reported age at first heterosexual intercourse was comparable in the two surveys: median ages of 17 (men) and 16 (women) among those aged 16-24. However, for some very sensitive questions there were lower levels of reporting in HSE-2010 than in Natsal-2: while the proportion reporting samesex in the last 5 years was similar (2-3\%), reporting of ever having same-sex was lower in HSE-2010 for men (2\% vs. 5\% in Natsal-2). Similarly, the mean number of opposite-sex partners reported in HSE-2010 was a little lower than in Natsal-2, particularly among men: 9.5 vs. 12.7, respectively, in contrast to 5.4 vs. 6.5, respectively, among women. Men in HSE-2010 were also slightly less likely to report being diagnosed with sexually transmitted infection(s) than in Natsal-2: $8.7 \%$ vs. $11.1 \%$, respectively; but this was reported by similar proportions of women: $12 \%$ in both surveys.

Conclusion The inclusion of questions on sexual behaviour in HSE-2010 has demonstrated the feasibility and utility of measuring sexual behaviour in general health surveys, albeit in less detail than in a survey focused on sexual behaviour such as Natsal. General health surveys such as the HSE provide a useful vehicle for monitoring sexual risk behaviour more frequently than is possible with decennial Natsal surveys. Health surveys should not shy away from also measuring sexual behaviour, especially in light of the associations between health status and sexual health outcomes of satisfaction, function and behaviour.

OP75 THE RELATIVE IMPORTANCE OF OBJECTIVE AND
SUBJECTIVE SOCIO-ECONOMIC AND SCHOOL-BASED
SOCIAL STATUS FOR ADOLESCENT HEALTH AND
SUBSTANCE USE

doi:10.1136/jech-2012-201753.075

H Sweeting, K Hunt, A Bhaskar. MRC Social and Public Health Sciences Unit, University of Glasgow, Glasgow, UK

Background Contrasting with findings from adults and children, most studies of adolescents find little variation in health according to conventional (objective) socio-economic status (SES) measures. Adolescent smoking is patterned by SES, but relationships between SES and drinking are weaker or non-existent. Subjective status captures perceptions of relative rank and may also be important for health. Most studies of health and subjective status focus on adults, use subjective SES and explore self-reported health rather than behaviours. However, subjective school-based status may be more important than subjective SES for health in adolescence. This study examines the relative importance of objective SES, subjective SES and school-based social status for adolescent self-reported health and health behaviours.

Methods Data were obtained via schools-based self-completion questionnaires in 2010 with follow-up in 2011 when 2,503 (85\% of baseline) $13-15$ year olds participated. Variables allowing derivation of family affluence were included in 2010; all other data were obtained in 2011. Scottish Index of Multiple Deprivation (SIMD) was derived via postcodes. Pupils rated subjective SES via the MacArthur Scale of Youth Subjective Social Status, a 10-rung ladder with the top representing 'the best off people in Scotland'. Seven ladders asked them to rate various aspects of their own status, compared to their school year-group. Questionnaires also asked about self-rated health, psychological distress (GHQ-12), smoking and drinking. Analyses suggested three subjective school-based social status dimensions: 'peer', 'scholastic' and 'sports'. Objective SES and all social status measures were each collapsed into three categories for inclusion in logistic regression analyses which were conducted on those with full data $(\mathrm{N}=1,819)$ on these measures.

Results Correlations between objective SES and all subjective status measures were weak. In preliminary multivariate logistic regression analyses, adjusted for gender and age, family affluence was not associated with health, smoking or drinking and deprivation was not associated with health. However, each subjective school-based status measure was associated with both health and behaviours. For example, odds (95\% confidence intervals) of fair/poor self-rated health among those 'low' compared with 'high': family affluence 1.1 (0.68-1.81); SIMD 1.23 (0.86-1.76); subjective SES 1.42 (0.972.08); subjective 'peer' status 1.73 (1.20-2.50); 'scholastic' 2.93 (2.01-4.27); 'sports' 2.93 (1.98-4.35). Odds of ever smoking among those low (vs. high): family affluence 1.43 (0.93-2.21); SIMD 2.28 (1.67-3.13); subjective SES 1.20 (0.84-1.71); 'peer' 0.30 (0.21-0.42); 'scholastic' 11.80 (8.05-17.29); 'sports' 2.00 (1.41-2.84).

Conclusion Subjective school-based social status is more important for adolescent health and substance use than either objective or subjective SES measures. 